\title{
Long-term evolution of the inclined geosynchronous orbit in Beidou Navigation Satellite System
}

\author{
Jingshi Tang ${ }^{\mathrm{a}, \mathrm{b}, *}$, Xiyun $\operatorname{Hou}^{\mathrm{a}, \mathrm{b}}$, Lin $\mathrm{Liu}^{\mathrm{a}, \mathrm{b}}$ \\ ${ }^{a}$ Key Laboratory of Modern Astronomy and Astrophysics, 83 School of Astronomy and \\ Space Science, Nanjing University, 163 Xianlin Avenue, Nanjing, 210023, China \\ ${ }^{b}$ Institute of Space Environment and Astrodynamics, Nanjing University, 163 Xianlin \\ Avenue, Nanjing, 210023, China
}

\begin{abstract}
China's Beidou Navigation Satellite System (BDS), unlike other navigation satellite systems, uses several inclined geosynchronous orbits (IGSO) to enhance the accuracy of regional or global navigation. In order to maintain a safe space environment in the vicinity of its operational orbit, it is necessary that the decommissioned satellites be well disposed of. To understand the underlying dynamics that affect the BDS IGSO, we study this problem from two aspects. In this paper, we first theoretically analyze the problem using the simplified models with 1 and 2 degrees of freedoms (1-/2-dof). Then we extensively investigate the numerically propagated orbits for 200 and 1000 years, applying the results from these simplified models and seeking proper explanations for the underlying dynamics. We especially focus on the eccentricity evolution, which is a major concern regarding the collision hazard. We expect to understand the underlying dynamics governing the long-term evolution of BDS IGSO and gain helpful insight into future disposal strategies.
\end{abstract}

Keywords: Beidou Navigation Satellite System (BDS), Inclined geosynchronous orbit (IGSO), Long-term evolution, Secular resonance

\footnotetext{
${ }^{*}$ Corresponding author

Email addresses: jstang@nju.edu.cn (Jingshi Tang), silence@nju.edu.cn (Xiyun Hou), lliu@nju.edu.cn (Lin Liu)
} 


\section{Introduction}

China has been deploying its own navigation satellite system, Beidou Navigation Satellite System (BDS), for over a decade. It is now operational for navigation service in China (see e.g. Jin et al. (2016) for BDS applications) and Asia-Pacific region and is due to be fully operational as a global navigation system by $2020 .^{1}$

Unlike other navigation satellite systems, BDS consists of the medium Earth orbit (MEO) with a period of about 12.89 hours and the 24-hour geosynchronous orbit. By the end of 2014, BDS includes six $55^{\circ}$-inclined $\mathrm{MEO}$, five $55^{\circ}$-inclined geosynchronous orbit (IGSO) and five equatorial geostationary Earth orbit (GEO). In this paper, we use these 5 BDS ISGO satellites as representative samples in our $\Omega_{0} \times \omega_{0} \operatorname{grid}^{2}$. According to the official BDS document, when fully deployed the complete constellation will consist of 22 MEO, 3 IGSO and 5 GEO satellites.

For the navigation satellites, it is important that they are properly disposed of at their end of life so they do not pose potential dangers to other working satellites. A common way to dispose the decommissioned GEO satellites is to put them in a higher graveyard orbit that guarantees no intersection with working orbits (see e.g. Anselmo and Pardini (2008)). Although this is also the current disposal strategy for many of the GPS satellites (Alessi et al.,, 2014) ${ }^{3}$, there have been plenty of researches (see e.g. Chao and Gick (2004); Rossi (2008); Pardini and Anselmo (2012)) showing that simply increasing orbital altitude of the decommissioned navigation satellites cannot prevent future intersection with the working orbit of its own constellation or other navigation systems.

Recently, Stefanelli and Metris (2015) theoretically analyzed the MEO satellites using a simplified 1 degree-of-freedom (1-dof) model and showed possible eccentricity $(e)$ evolution under this 1-dof model. They show that

\footnotetext{
${ }^{1}$ According to the document: BeiDou Navigation Satellite System - Signal In Space Interface Control Document Open Service Signal (Version 2.0), by China Satellite Navigation Office released in December 2013.

${ }^{2}$ As of April 2016, there are totally 8 BDS IGSO satellites in orbit. According to online sources (https://en.wikipedia.org/wiki/List_of_Beidou_satellites, accessed on 29 April, 2016), 7 of them are operational and the most recent one is still in commissioning.

${ }^{3}$ Exceptions are NAVSTAR 2 and 6, which were maneuvered respectively about $90 \mathrm{~km}$ and $250 \mathrm{~km}$ below at the end of life.
} 
under some proper initial conditions, $e$ may increase to as large as 0.8, posing dangers to navigation satellites in the commonly used 12-hour MEO. Particularly, they showed with their simplified model that, compared to the classic Hughes (1980) results which claimed resonance at the inclination of $56^{\circ} .1$ due to the argument $2 \omega+\Omega$, the actual resonance inclination for $2 \omega+\Omega$ at which resonance is to occur would shift for Galileo MEO considering the contribution from the long-period term. Besides that, Rosengren et al. (2015) studied the MEO navigation satellites under secular resonance and resonance overlap and suggested chaos in those orbits under lunar perturbation, resembling the chaotic behaviors in the Solar System.

A major reason to the increase in the orbit eccentricity of the MEO navigation satellites is closely related to their $55^{\circ}$ orbit inclinations. Determined mainly by the initial phases of the right ascension of ascending node (RAAN, $\Omega$ ), their mean inclinations can be close to the resonance inclination. The mean inclination in this work is defined in terms that all the period terms including short-period (dependent on the fast-varying arguments such as the mean anomaly $M$ ) and long-period (dependent on the slow-varying arguments such as $\Omega$ ) terms are removed from the osculating elements ${ }^{4}$. The problem for BDS is that for its $55^{\circ}$-inclined IGSO, how would its long-term orbital evolution become. Earlier works (see e.g. Tang and Liu (2015); Zhao et al. (2015)) already show that for the 5 BDS IGSO (denoted as \#1 \# \#5 hereinafter), although they have close semi-major axes, eccentricities and inclinations $(a, e, i)$, they have quite different long-term evolutions, all owing to the different initial $\Omega$ and arguments of perigee $(\omega)$.

In our previous work (Tang and Liu, 2015), we ran a batch test over the grid of $\left(\Omega_{0} \times \omega_{0}\right) \in(2 \pi \times 2 \pi)$ with a resolution of $2^{\circ} \times 2^{\circ}$, numerically integrated for 200 years. Besides the lifetime contours therein, we also obtain maximum and minimum eccentricity contours, as shown here in Fig. 1. Although these lifetime and eccentricity contours are obtained using initial osculating $a, e, i$ of IGSO \#1, we showed that they are generally applicable for other IGSO satellites as well.

In this work, we focus on the special features in the eccentricity contours under the secular resonance, the commensurability between the slow-varying

\footnotetext{
${ }^{4}$ One can choose to use various analytical formulas to remove the period terms. As far as the long-period perturbations from $J_{2}$ and lunisolar gravitation are concerned, the equations of motion are listed in the supplementary materials and the perturbation solutions can be easily obtained by quadrature. They can also be found in Liu (2000).
} 

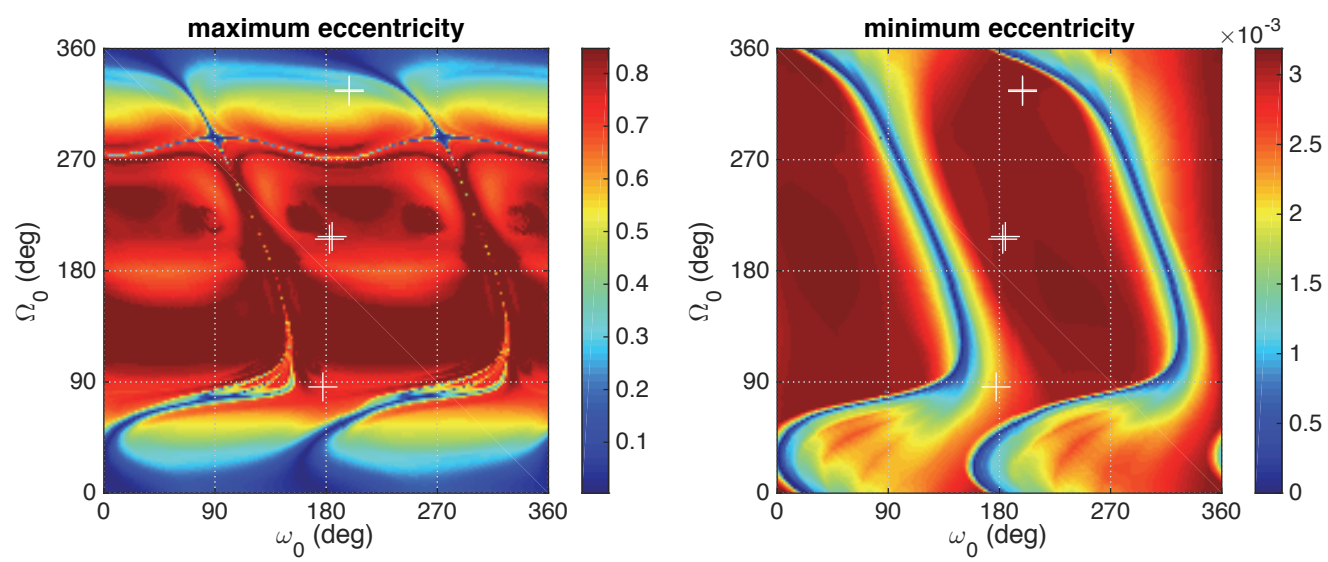

Figure 1: The contour of the maximum eccentricity (left) and minimum eccentricity (right) in 200 years with respect to different initial phases: $\omega_{0}$ and $\Omega_{0}$. The tests are carried out based on IGSO \#1's initial elements $\left(a_{0}, e_{0}, i_{0}\right.$ and mean anomaly $\left.M_{0}\right)$. The BDS IGSO satellites are annotated with the white plus signs (top ones for \#2 and \#5, middle ones for \#1 and \#4 and bottom one for \#3).

arguments $\Omega_{0}$ and $\omega_{0}$ (see e.g. Murray and Dermott (1999)). The motivation of this work is to provide useful insight for BDS decision makers and mission planners regarding the IGSO disposal strategy and the implication. In Section 2, we introduce the models we use in the work, including the analytical equations and force models. Then we theoretically study the simplified models for BDS IGSO in Section 3. Here we specifically focus on the secular resonance that shapes the long-term eccentricity evolution. In addition to the secular terms, the long-period contribution from the lunisolar perturbation is included in the equations of motion to better determine the equilibria and the associated conditions (in terms of $e$ and $i$ combinations). In Section 4 , we investigate the long-term evolution of BDS IGSO in the real force models. We numerically propagate the orbits for 200 and 1000 years. With the help from the theoretical results, we can explain the underlying dynamics and understand the relation between the eccentricity and the initial phases (Fig. 1). Summary and discussions are organized in Section 5.

\section{Models and methods}

Our investigation includes extensive theoretical analysis and numerical propagation. The analytical approaches provide us with fundamental qualitative features of the system, and it is used to help us understand the results 
from the numerical propagation in the real force model. The models and methods for the analytical and numerical approaches are introduced here.

\subsection{Analytical equations for the long-period terms}

For our theoretical analysis, we use a simplified model to account for the long-period term from which the secular resonances rise.

The simplified model in this work includes major perturbations namely the $J_{2}$ geopotential term and the lunisolar gravitation. For the latter, we only take the largest term in the expansion, which is the $P_{2}$ term in the Legendre polynomials.

The equations of motion for the Keplerian elements can be found in Kozai (1959). For the lunisolar gravitation, we adopt the expansion introduced in Kozai (1973). The perturbing accelerations for the Keplerian elements were also provided therein, but we slightly modify the forms of the equations, as are shown in Liu (2000).

After double average over the mean motions of the satellite and the perturbing body and also assuming circular orbits for the Moon and the Sun, we are able to obtain the long-period acceleration for the eccentricity

$$
\begin{aligned}
& \left\{\begin{aligned}
\frac{d e}{d t} & =\sum_{k=1}^{2}\left(\frac{3}{2} \beta_{k} a^{3}\right)\left(5 e \sqrt{1-e^{2}}\right) n H_{1} \\
H_{1} & =\frac{1}{16}\left[\frac{1}{2}(1-\cos i)^{2} K_{1}+\frac{1}{2}(1+\cos i)^{2} K_{2}+\sin ^{2} i K_{3}\right.
\end{aligned}\right. \\
& \left.\quad+2 \sin i(1-\cos i) K_{4}+2 \sin i(1+\cos i) K_{5}\right]
\end{aligned}
$$

where the sum over $k$ stands for the perturbing gravitation from the Moon $(k=1)$ and the Sun $(k=2)$ and the parameters for the perturbing body is denoted by prime $\left(^{\prime}\right) . \theta$ is the elongation between the ascending nodes of the satellite and the perturbing body, i.e. $\theta=\Omega-\Omega^{\prime}$ and $\beta_{k}=m_{k}^{\prime} /\left(r_{k}^{\prime}\right)^{3}$.

Eq. (1) suggests that for the eccentricity there are five angular arguments that can potentially contribute to the secular resonance. Hughes (1980) enumerated all possible resonances on the Earth satellites from a general 
astrodynamical perspective, while Chao and Gick (2004) first pointed out the implication of these 5 arguments on the $55^{\circ}$-inclined navigation satellite constellations. To further simplify the problem, when we theoretically discuss the dynamics in this paper, we ignore the fact that the lunar orbit oscillates around the ecliptic with an amplitude of about $5^{\circ}$ and simply take the ecliptic as the mean lunar orbit.

For the equations of motion of the simplified models, the complete perturbing accelerations for $e, i, \Omega$ and $\omega$ are needed to account for the secular and long-period accelerations from $J_{2}$ and lunisolar gravitation. To save space, the complete equations are listed in the supplementary materials.

It needs to be mentioned that throughout this paper, we use normalized units commonly used in celestial mechanics and orbital dynamics. The unit length $[L]$ is the equatorial radius of the Earth reference ellipsoid $a_{e}$, and the unit mass $[M]$ is the mass of Earth $M_{e}$. The unit time $[T]$ is defined as $[T]=\sqrt{a_{e}^{3} / G M_{e}}$ such that the geocentric gravitational constant $\mu=G M_{e}$ is 1. For the BDS IGSO satellites, they have close semi-major axes around 6.61 and this value will be used in the following analysis.

\subsection{Numerical propagation in the real force model}

Our goal is focused on the eccentricity evolution in the real force model. For that, the equations of the motion of the full system is integrated using a 7th-order Runga-Kutta-Fehlberg integrator RKF7(8) (Fehlberg, 1968). It is capable of adaptive stepsize control and we limit the truncation error per step at $10^{-13}$. This is a commonly used numerical method in many areas and commercial softwares (e.g. the HPOP propagators in STK). For the long-term propagation, Liu et al. (1994) compared various RKF methods and some variants to the symplectic method, regarding the propagation of various Earth satellite orbits. The tests therein suggest that for the propagation of $10^{4} \sim 10^{5}$ orbital periods in our work, the RKF7(8) method can serve well enough without any noteworthy impact on the results or the conclusions.

The orbit is described by the following nonsingular orbital elements that eliminates the $e=0$ singularity

$$
a, i, \Omega, \xi=e \cos \omega, \eta=e \sin \omega, \lambda=M+\omega .
$$

The perturbations considered in orbit propagation include the geopotential up to degree/order 10 using the EGM model, lunisolar gravitation using the ephemeris of JPL DE406 and the solar radiation pressure assuming a 
moderate area-to-mass ratio of $0.01 \mathrm{~m}^{2} / \mathrm{kg}$. We do not consider the atmosphere drag even when the BDS IGSO gets highly eccentric and could possibly reenter the atmosphere at its perigee. The time at its perigee is so short that the drag could hardly affect the orbit. This assumption agrees with the real observations for the Sino-Euro DoubleStar satellites (Tang et al., 2012), both of which are in highly eccentric orbits. The orbit end-of-life is assumed when the satellite hits the Earth surface and for BDS IGSO this critical eccentricity $e_{c}$ is around 0.85 .

\section{Theoretical analysis}

1-dof model is usually applied to analyzing the individual resonances in the navigation satellite system (see e.g. Rossi (2008); Stefanelli and Metris (2015)). To accommodate the 1-dof model to our problem, we use the following conjugated elements which suits all the five resonance arguments in Eq. (1)

$$
\begin{array}{ll}
\text { angle : } q=\kappa \omega+\nu \Omega & , \Omega \\
\text { action : } & p=\frac{G}{\kappa} \quad, K=H-\frac{\nu}{\kappa} G .
\end{array}
$$

where $G=\sqrt{\mu a\left(1-e^{2}\right)}$ and $H=G \cos i$ are the Delaunay elements. In the scope of this paper, we have $\kappa=2$ while $\nu$ being any integer between -2 and 2. When any one of the arguments is in the state of resonance, $\Omega$ can be eliminated and the system is reduced to 1 degree of freedom.

To save space, the common procedures of processing the 1-dof model is not repeated in the main article, but is provided in the supplementary materials for references. We recommend the readers to refer to the previous papers (e.g. Stefanelli and Metris (2015)) for common knowledge and also to the supplementary materials for relevant details.

The equilibria from the 1-dof model are summarized in Table 1. Given the background of this work, only the prograde orbits with $i \in[0, \pi / 2)$ are considered, while the retrograde orbits can be likewise analyzed. It should be noted that these results are obtained assuming small eccentricity (i.e. $e^{2} \approx 0$ ), and they can be quite different when $e$ is large. Fig. 2 shows the distribution of the equilibria of the arguments in the 1-dof model for $e_{0} \in[0,0.8]$. It is clear that not all the equilibria always exist. Specifically, $q_{0}=0$ does not exist until $e$ is over 0.37 .

Although BDS IGSO satellites are initially inclined approximately $55^{\circ}$, their mean inclinations can differ due to their different initial phase $\Omega_{0}$. One 
Table 1: Summary of the resonance arguments for BDS IGSO, including the resonance inclinations and their stabilities. The values are computed at $e=0.1$. Should the equilibrium exist, 'S' and 'U' denote stable (center) and unstable (saddle) respectively.

\begin{tabular}{ccccccc}
\hline Resonance & \multicolumn{2}{c}{ Resonance inclination } & & \multicolumn{2}{c}{ Stability } \\
\cline { 2 - 3 } \cline { 5 - 6 } argument & 0 & & & & 0 & $\pi$ \\
\hline$q_{0}=2 \omega$ & - & $52^{\circ} .86$ & & - & $\mathrm{S}$ \\
$q_{1}=2 \omega-2 \Omega$ & $73^{\circ} .63$ & $72^{\circ} .80$ & & $\mathrm{U}$ & $\mathrm{S}$ \\
$q_{2}=2 \omega+2 \Omega$ & $49^{\circ} .37$ & $43^{\circ} .09$ & & $\mathrm{~S}$ & $\mathrm{U}$ \\
$q_{9}=2 \omega-\Omega$ & $75^{\circ} .92$ & $64^{\circ} .95$ & & $\mathrm{U}$ & $\mathrm{S}$ \\
$q_{10}=2 \omega+\Omega$ & $45^{\circ} .48$ & $70^{\circ} .75$ & & $\mathrm{~S}$ & $\mathrm{~S}$ \\
\hline
\end{tabular}

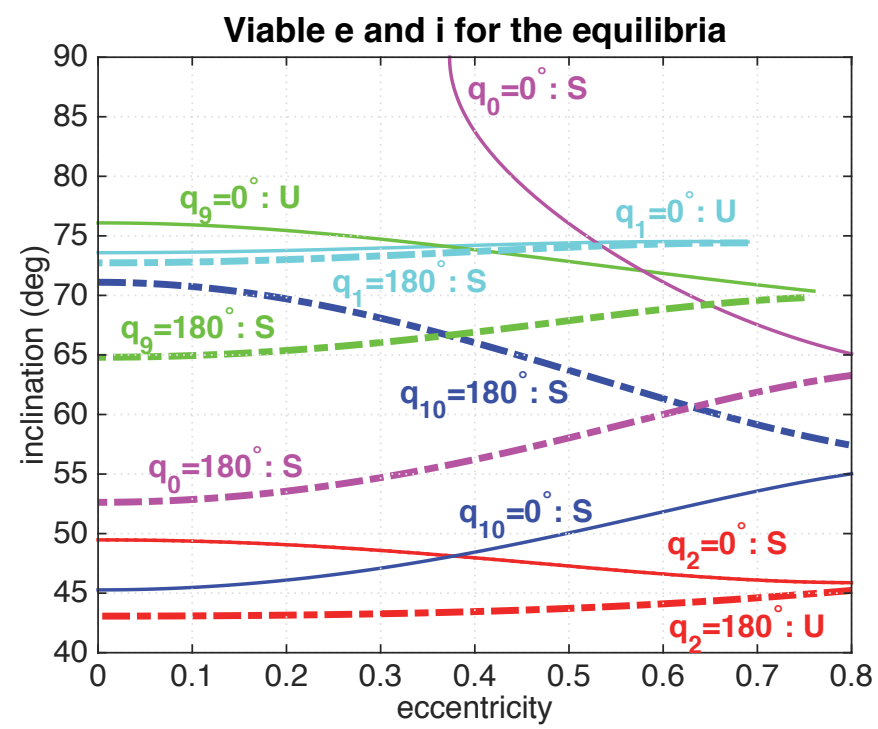

Figure 2: Distribution of the equilibria with respect to initial osculating $e$ and $i$, together with their stabilities. 'S' and ' $U$ ' denote stable and unstable equilibria respectively, while the solid and dashed lines are used for the $q_{j}=0$ and $q_{j}=\pi$ equilibrium points respectively. 

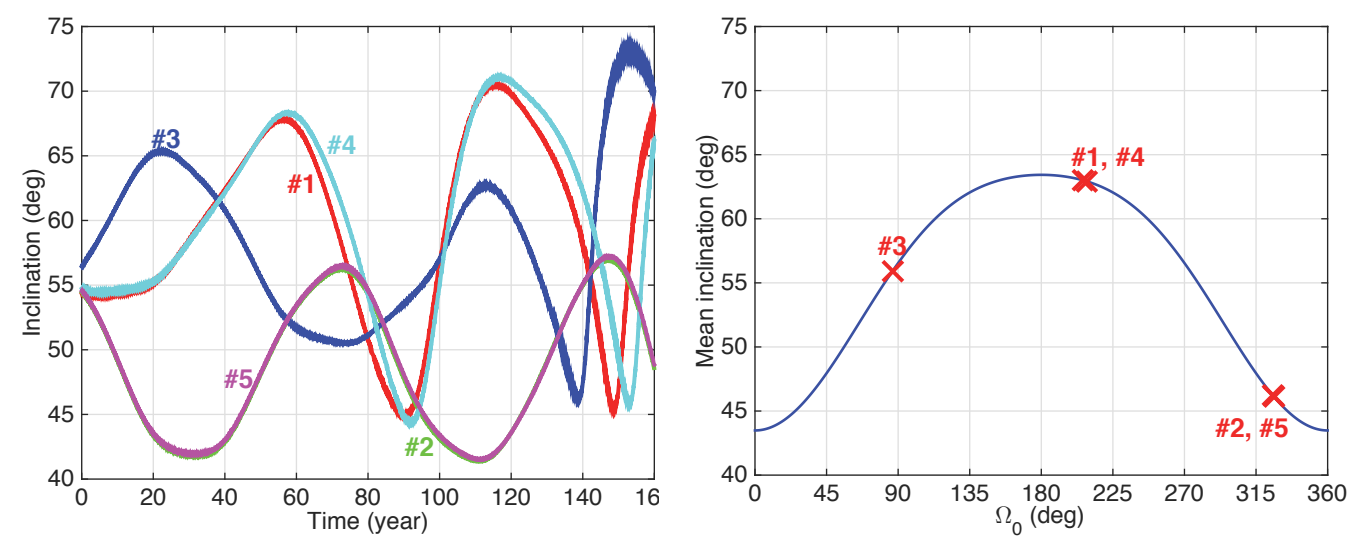

Figure 3: Left: the temporal inclination profiles of the five relevant BDS IGSO satellites, all of which are numerically propagated using the full force models. One can estimate the mean inclination of each satellite from the time series; Right: the mean inclination with respect to $\Omega_{0}$, starting from the osculating inclination of $55^{\circ}$ and computed by removing the analytical long-period term (see the supplementary materials). The five BDS IGSO satellites are marked by their numbers.

can choose to numerical propagate the BDS IGSOs and observe the temporal inclination profiles (left frame of Fig. 3) or to analytically remove the longperiod terms and obtain the mean inclinations (right frame of Fig. 3). The results from the numerical and analytical approaches are generally consistent, and it is not difficult to find that $q_{1}=0$ or $q_{1}=\pi$ has resonance inclinations (Table 1) too large to affect the BDS IGSO.

Stefanelli and Metris (2015) has shown that for the $q_{10}$ resonance in the Galileo system, the phase diagram of the 1-dof model resembles that of a simple pendulum. This is also true for the BDS IGSO and for other resonance arguments besides $q_{10}$. Selected phase diagrams for the relevant resonance arguments, as well as the eccentricity time series, are shown in the supplementary materials. It is to be mentioned that the 1-dof model depends on the parameter $K$, which remains constant $\sqrt{\mu a_{0}\left(1-e_{0}^{2}\right)}\left(1-\frac{\nu}{\kappa} \cos i_{0}\right)$ with time. With different $K$, the equilibrium (location/condition/stability/number) of the same argument may differ accordingly. We present the bifurcations of $q_{9}$ and $q_{10}$ in the supplementary materials and it is interesting to note the $q_{10}$ 's bifurcation, where it is obvious that in certain situations (around $K_{10}=0.05$ ) the system has three equilibria ( 2 centers and 1 saddle). Nevertheless two of them occur for $e>0.9$ which is well beyond the critical eccentricity and cannot be observed in the actual BDS IGSO evolution. 
Although the 1-dof model shows us possible equilibria of the system, it can hardly represent the actual orbit evolution. To have a brief understanding of the possible situations we may encounter when practically analyzing the BDS IGSO, we allow the system to have a second degree of freedom and see how the orbits evolve in the 2-dof model. The practical system is of course more complicated than the 2-dof model studied in this section, in terms that many resonant terms can simultaneously contribute. In order to better illustrate the effects of chaos by the interplay of different resonances, here we choose the simpler 2-dof model, while the full force model will be considered in the next section. Given the purpose of this study, we do not intend to tackle the 2-dof problem analytically, but simply investigate it via a numerical approach. To save space, we leave the calculation details and results in the supplementary materials, while summarize below the major features of the 2-dof model other than the simple libration or circulation in the 1-dof model.

1. Around the original stable equilibrium of the 1-dof system, the 2-dof system (regarding the argument) could transit between libration and circulation;

2. In our case, the chaotic motion in the 2-dof model can always lead to large orbit eccentricity. As a result, the equilibrium position obtained in case of small eccentricity can change. A case in point is shown in the supplementary material, that for inclinations between $55^{\circ} \sim 65^{\circ}$, the equilibrium position of $q_{10}$ changes from 0 (Table 1 ) to $\pi$;

3. The system would transit between libration of different arguments alternately. This resembles the resonance transitions in the Solar System small bodies, suggesting that BDS IGSO satellites may transit between secular resonances in long-term evolution.

\section{Actual BDS IGSO long-term evolution}

In this section, we discuss the actual BDS IGSO long-term evolution in real force model using the numerical integrator introduced in Sec. 2.2. All the figures in this section are based on these numerical results, unless otherwise stated. We recall the 200-year eccentricity contours (Fig. 1) and extract the patterns in the left frame of Fig. 4.

We choose 4 areas with special patterns. Area \#I and \#II are distinguished by their $\Omega_{0}$ values (see also the left frame of Fig. 1). Area \#I is 

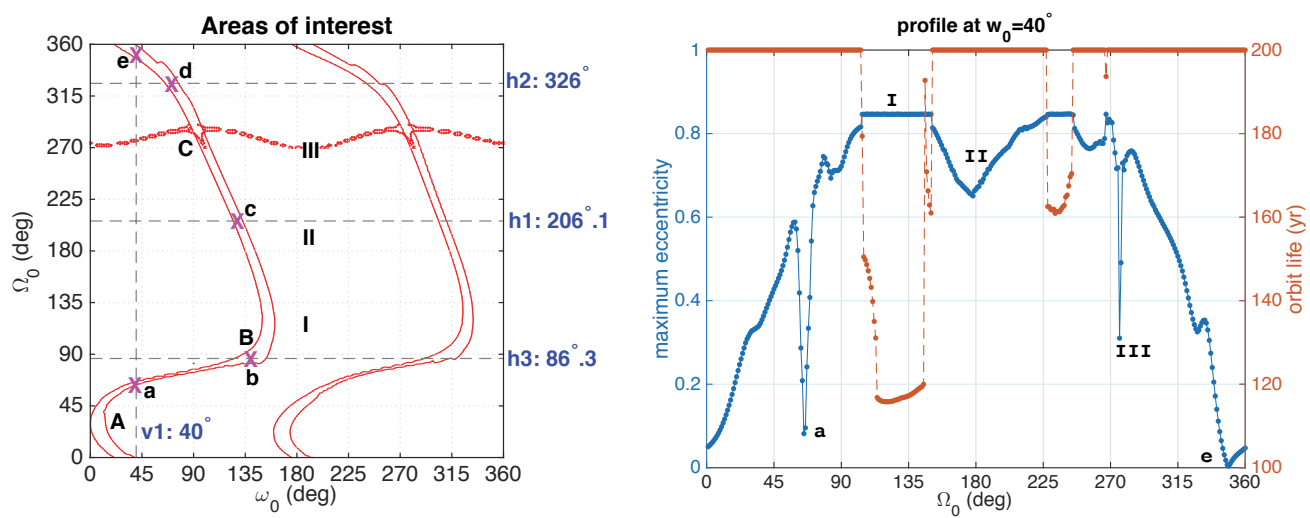

Figure 4: Left: Special patterns extracted from the eccentricity contours in Fig. 1. Due to the symmetry of $\omega \in[0, \pi)$ and $\omega \in[\pi, 2 \pi)$, we focus on the left half $(\omega \in[0, \pi))$. We divide the contour into four major areas of interest and also choose four vertical or horizontal profiles to investigate the dynamics. We use the roman numbers for the areas (Area \#IV is the stripe), gray lines for the profiles, capital letters for the turnings along the stripe and small letters (in bold to be distinguishable from the orbital elements) with magenta ' $x$ ' for the intersection points of the stripes and the profiles. Right: The maximum eccentricity (left $y$-axis of blue line with dots) and the orbital lifetime (right $y$-axis of red dash line with dots) in 200 years with respect to $\Omega_{0}$, along the profile of $\omega_{0}=40^{\circ}$. The letters $(\boldsymbol{a}$, $e)$ and numbers (I, II, III) show the intersections of the profile with corresponding areas.

defined approximately between $90^{\circ}$ and $180^{\circ}$ where test satellites decay in 200 years, whereas Area \#II is defined approximately between $180^{\circ}$ and $270^{\circ}$ where many of them survive the decay. Area \#III is a narrow line just around $270^{\circ}$ and is only observable in the $e_{\max }$ contour. The last but most interesting area is the stripe (Area \#IV) extending from bottom to top with some turnings.

To investigate the details, we choose 4 profiles that have the same $\omega_{0}$ or $\Omega_{0}$ (left frame of Fig. 4). They are 1 vertical profile $\mathbf{v} \mathbf{1}$ at $\omega_{0}=40^{\circ}$ and 3 horizontal profiles $\mathbf{h} \mathbf{1}, \mathbf{h} \mathbf{2}, \mathbf{h} \mathbf{3}$ at $\Omega_{0}=206^{\circ} .1,326^{\circ}, 86^{\circ} .3$ respectively. The vertical profile is chosen so as to intersect or be near to as many specific patterns as possible. The horizontal profiles are chosen at the $\Omega_{0}$ of three of BDS IGSO satellites (\#1 \#3, and hence the names $\mathbf{h} \mathbf{1} \sim \mathbf{h} \mathbf{3})$.

In the following sections, we discuss the underlying dynamics of the eccentricity contours by areas, using the auxiliary profiles. The turning points and intersection points are to be referred to wherever needed. 


\subsection{Secular resonance in 200 years}

The $e_{\max }$ value and the orbital lifetime along the auxiliary profile $\mathbf{v} \mathbf{1}$ are plotted in the right frame of Fig. 4, which is later used to assist in investigating the eccentricity contours of BDS IGSO.

In this section, we investigate by areas the 200-year dynamics for the BDS IGSO configurations. First, we note that despite Areas \#III and \#IV, the eccentricity shows a large-scale major dependence on the initial phase of $\Omega_{0}$, which can be seen around $\Omega_{0}=0$ in Fig. 4. This dependency is closely related to the resonance argument of $q_{10}=2 \omega+\Omega$. As the 1-dof model shows (Table 1 ), the resonance inclination of $q_{10}=0$ is approximately $45^{\circ} .5$, which can only be accessible when $\Omega_{0}$ is near 0 . The libration of $q_{10}$ around the stable equilibrium of $q_{10}=0$ helps to suppress the eccentricity in the long-term dynamics and starting further from $\Omega_{0}=0$ results in larger $e_{\max }$. The details can be seen when we investigate the individual areas.

\subsubsection{Area \#IV}

We start from Area \#IV - the stripe. This area stands out due to the relatively small eccentricity maximum and minimum $\left(e_{\min }\right)$ over 200 years. The stripe is divided into three segments by the turnings marked $A, B, C$ and is discussed one by one accordingly.

\section{Segment $A-B$}

This segment can be investigated using its intersection with the $\mathbf{v} \mathbf{1}$ profile at point $\boldsymbol{a}$. Around the intersection, we choose 4 tests along the profile and plot the time series of the eccentricity, inclination and arguments $q_{0}, q_{10}$ in Fig. $5^{5}$. We see that the $A-B$ segment of the stripe is where $q_{0}$ is able to librate around its stable equilibrium of $q_{0}=\pi$. When the equilibrium condition (mainly dependent on $i$ in case of small eccentricity) is satisfied and the arguments $\Omega, \omega$ start from the appropriate initial phases, the $q_{0}$ argument is able to librate for about 80 years and the eccentricity can be effectively suppressed in the duration.

The system exits from the $q_{0}$ equilibrium state after 80 years, owing to various perturbations, yet we can see that it transits to the an equilibrium state of $q_{10}$. We note that in Fig. $5 q_{10}$ seems to be librating somewhere

\footnotetext{
${ }^{5}$ In case the readers would like to better investigate the time series, we provide additional raster images for all individual time series in this section. The raster images are exported in PNG format with 150 pixels per inch and are uploaded in a single package.
} 

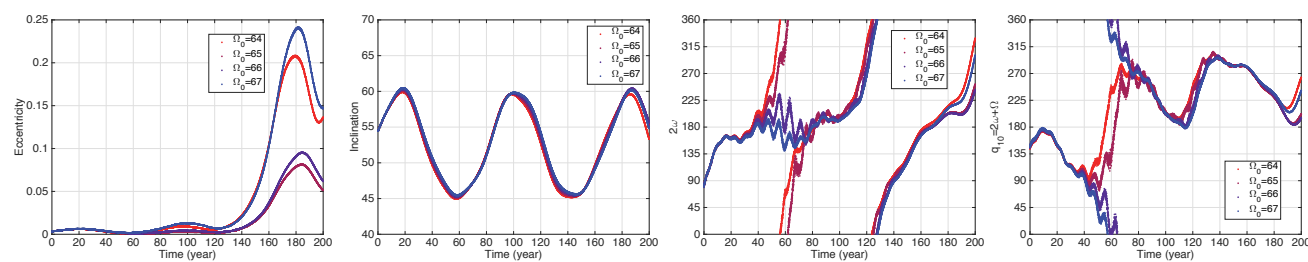

Figure 5: Time series of tests around the intersection point $\boldsymbol{a}$, for investigation of the segment $A-B$ in Area \#IV.

around $\sim 250^{\circ}$ which is not necessarily its stable equilibrium, but if we could observe the time series for an even longer time, we would find that the $q_{10}$ argument librates around $q_{10}=0$ from a long-term perspective. This is also why the eccentricity can remain relatively small although $q_{10}$ is seemingly not librating around its stable equilibrium. Details on the $q_{10}$ time series are to follow in the next section regarding the orbit evolution in a millennium.

It is to be mentioned that although the mean inclination should be generally symmetric on both sides of $\Omega_{0}=\pi$, we cannot expect the stripe to bear such symmetry. The reason to the asymmetry of the stripe lies in the fact that the stripe essentially results from the resonances of various different arguments. For resonance of each argument, its occurrence requires appropriate initial phase and this does not guarantee symmetry of either $\Omega_{0}$ or $\omega_{0}$ whatsoever.

\section{Segment $\boldsymbol{C}$ - $\boldsymbol{A}$}

To investigate this segment, we use the intersections with the profiles $\mathbf{h} \mathbf{2}$ and $\mathbf{v} \mathbf{1}$ at points $\boldsymbol{d}$ and $\boldsymbol{e}$ respectively. The time series for $\boldsymbol{d}$ are plotted in Fig. 6 and $\boldsymbol{e}$ in Fig. 7. Time series at both points suggest that along the $C$ - $A$ segment $q_{2}$ librates around its stable equilibrium of $q_{2}=0$ for about $60 \sim 80$ years. In the duration, the eccentricity can be suppressed, which makes it prominent in the $e_{\min }$ contour. The mean inclination, as can be seen in Figs. 6 and 7, is around $49^{\circ}$ which satisfies the equilibrium condition for $q_{2}=0$. The $C$ - $A$ segment outlines the area of the resonance of $q_{2}$, where the initial $(e, i)$ satisfies the equilibrium condition and the initial phases of $(\Omega, \omega)$ are appropriate to start the libration.

Similar to the $A-B$ segment, the system will transit to the equilibrium state of $q_{10}=0$ after exiting from the $q_{2}=0$ equilibrium. The $q_{10}=0$ equilibrium helps to keep the eccentricity from increasing to large values. Over two centuries, the $e_{\max }$ value is below 0.45 for point $\boldsymbol{d}$ and below 0.03 

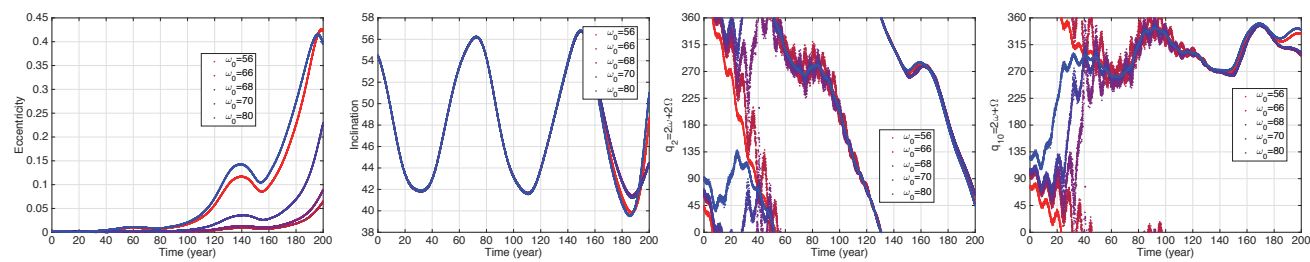

Figure 6: Time series of tests around the intersection point $\boldsymbol{d}$, for investigation of the segment $C$ - $A$ in Area \#IV.
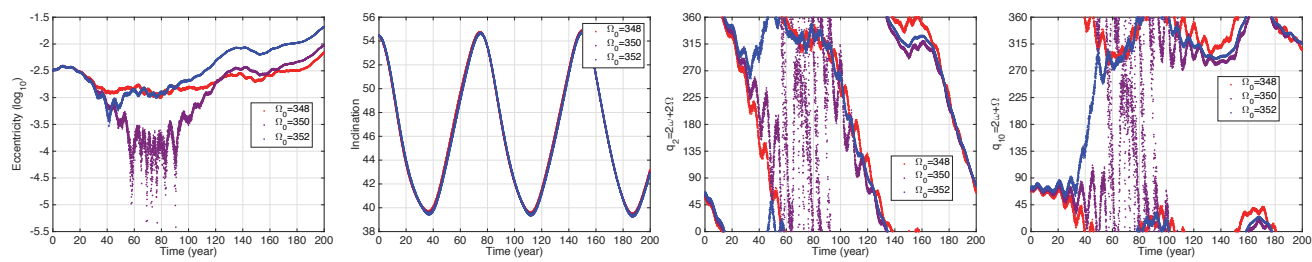

Figure 7: Time series of tests around the intersection point $\boldsymbol{e}$, for investigation of the segment $C$ - $A$ in Area \#IV.

for $\boldsymbol{e}$. The reason that $e_{\max }$ in the period of 200 years at $\boldsymbol{e}$ is smaller than its value at $\boldsymbol{d}$ is that $q_{10}$ librates more closely to its stable equilibrium at $\boldsymbol{e}$ than at $\boldsymbol{d}$. As seen from Fig. 4 (right frame), point $\boldsymbol{e}$ has the smallest $e_{\max }$ along the v1 profile, and this could be where $q_{10}$ librates closest to the stable equilibrium of $q_{10}=0$ in the period.

\section{Segment $B-C$}

The dynamics behind the segment $B-C$ is investigated using the intersections with profiles $\mathbf{h} \mathbf{3}$ and $\mathbf{h} \mathbf{1}$ at points $\boldsymbol{b}$ (Fig. 8) and $\boldsymbol{c}$ (Fig. 9) respectively. Along the stripe, argument $q_{9}$ can maintain in libration around its stable equilibrium of $q_{9}=\pi$ for about 50 years, before transiting into circulation. Just as in segment $A-B$ for $q_{0}$ and $C$ - $A$ for $q_{2}$, certain combinations of $(e, i)$ and $(\Omega, \omega)$ have to be satisfied so that the equilibrium condition and the initial phases are appropriate for $q_{9}$ to librate.

However, unlike in segments $A-B$ and $C$ - $A$, here we do not observe that the system transits into the equilibrium of $q_{10}$ whatsoever. It is natural since the mean inclination is now too far away from the equilibrium condition for $q_{10}=0$. After the system exits the equilibrium state of $q_{9}=\pi$, there is no mechanism to constrain the eccentricity and it can increase to large values. In some of the tests the orbit hits the Earth surface within two centuries. It is therefore worth to mention that the $B-C$ segment is prominent in the 

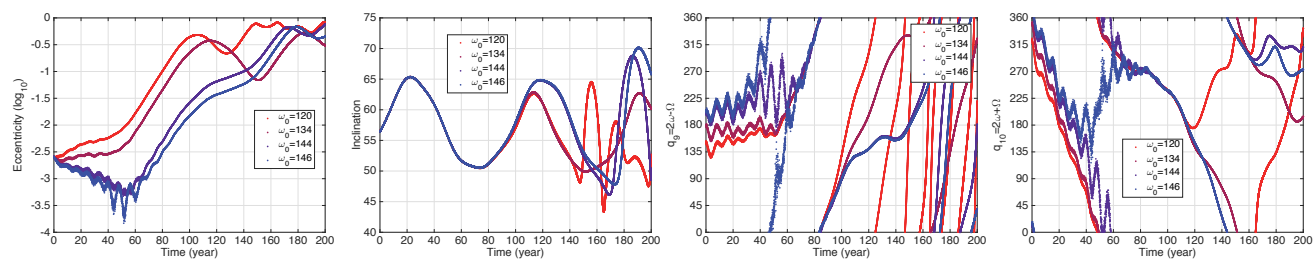

Figure 8: Time series of tests around the intersection point $\boldsymbol{b}$, for investigation of the segment $A-B$ in Area \#IV.
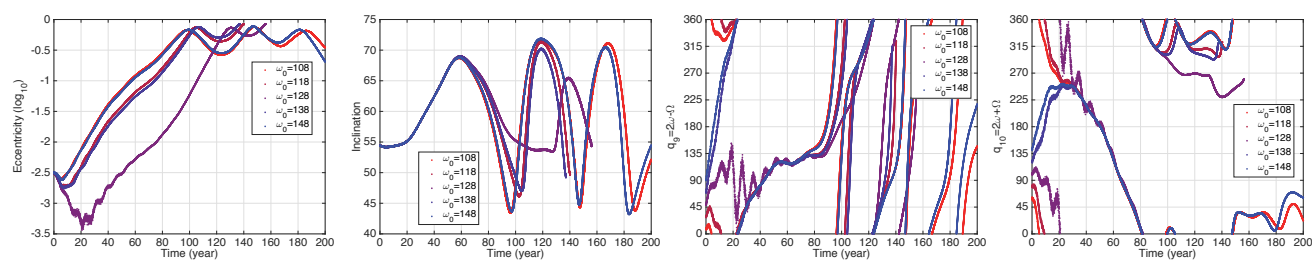

Figure 9: Time series of tests around the intersection point $\boldsymbol{c}$, for investigation of the segment $A-B$ in Area \#IV.

$e_{\text {min }}$ contour, just as the $A-B$ and $C-A$ segments. The small $e_{\min }$ is due to the libration of the resonance argument $q_{9}$ in the first few decades. While in the $e_{\max }$ contour, the values in segment $B-C$ is not so small compared to the other segments. The reason is the disappearance of the equilibrium state of $q_{10}$, which effectively suppresses the eccentricity from increasing too much in the other two segments.

Summarizing the investigations on the segments of the stripe (Area \#IV), we can draw some conclusions regarding the underlying dynamics that shape the stripe.

- The stripe is where secular resonances take place. The resonances of arguments $q_{0}, q_{2}$ and $q_{9}$ help to suppress the minimum eccentricity in the first few decades;

- The equilibrium condition and the initial phase need to be satisfied so that the corresponding resonance can occur. For the equilibrium condition, the mean inclination is essentially related to the initial value of $\Omega_{0}$. Dependencies on both $\Omega_{0}$ and $\omega_{0}$ determine the location of the stripe in the $\omega_{0} \times \Omega_{0}$ grid;

- The equilibrium state of $q_{10}$ is not rare in the grid. Actually it can be 

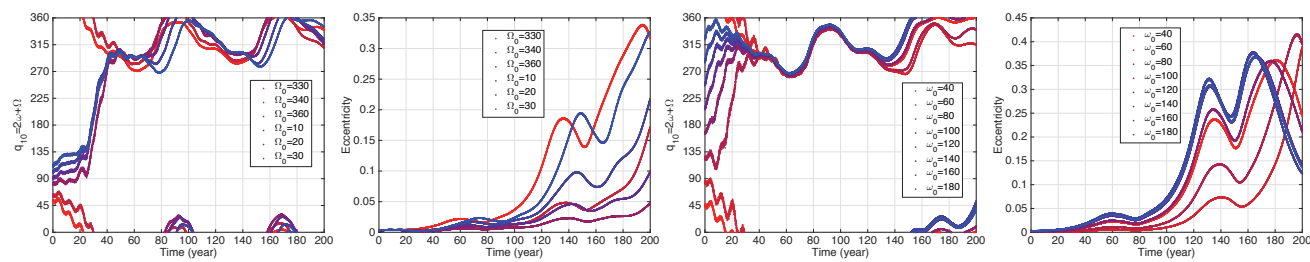

Figure 10: Time series of $q_{10}$ of $\mathbf{v} \mathbf{1}$ (1st), $e$ of $\mathbf{v} \mathbf{1}$ (2nd), $q_{10}$ of $\mathbf{h} \mathbf{2}$ (3rd) and $e$ of $\mathbf{h} \mathbf{2}$ (4th).

commonly seen on both sides of $\Omega_{0}=0$ and the reason, as explained before, is the satisfaction of the equilibrium condition (mainly $\bar{i}$ for small eccentricity) for $q_{10}=0$. We choose 3 tests on either side of $\Omega_{0}=0$ along the $\mathbf{v} \mathbf{1}$ profile and 8 tests between 0 and $\pi$ along the h2 profile. Fig. 10 shows the time series of the $q_{10}$ argument and the eccentricity. It is clear that the equilibrium state of $q_{10}$ helps to constrain $e_{\max }$, and this is why in the $e_{\max }$ contour, segments $A-B$ and $C-A$ stand out while segment $B-C$ does not;

- The stripe has three turnings. These turnings are where the contributing resonance argument changes, namely $q_{2}$ upward to $q_{0}$ at $A, q_{0}$ upward to $q_{9}$ at $B$ and $q_{9}$ upward to $q_{0}$ at $C$. These arguments generally dominate the system for first few decades and the dynamics well match the theoretical 1-dof model in the previous section. Besides, the transitions between different resonance arguments also agree with the behaviors of the 2-dof models.

\subsubsection{Area \#III}

This area appears as a narrow 'line' that is almost solely dependent on $\Omega_{0}$, and it can only be seen in the $e_{\max }$ contour. Given the shape of this area, it is clearly attributed to the dynamics of argument $q_{0}$. Fig. 11 shows the time series at the intersection of Area \#III and the $\mathbf{v} \mathbf{1}$ profile, where the $q_{0}$ argument is able to stay around its stable equilibrium $q_{0}=\pi$ over 200 years. It keeps the eccentricity not more than 0.3 , but since the $e_{\text {min }}$ value is simply about its initial eccentricity, this area is not observable in the $e_{\text {min }}$ contour. As $q_{0}$ stays around $q_{0}=\pi$ in this particular case, all other arguments including $q_{10}$ circulate throughout $[0,2 \pi)$. 

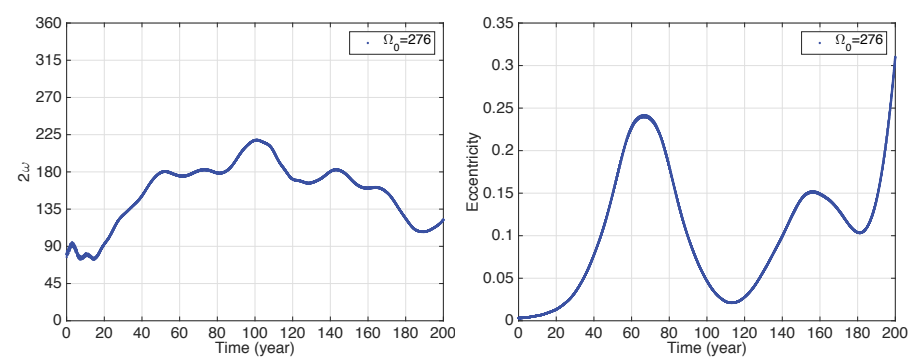

Figure 11: Time series of $q_{0}$ and $e$, at the intersection of Area \#III and the profile v1.

\subsubsection{Areas \#I and \#II}

It has been shown that outside Areas \#III and IV, there is no major resonance except for the argument $q_{10}$. The dependency on $\Omega_{0}$ has been briefly explained at the beginning of this section and numerically presented in Fig. 10. Therefore, it is reasonable to see that starting further from $\Omega_{0}=0$, the test orbit should have larger $e_{\max }$ value due to the gradual failure of the $q_{10}$ equilibrium, and at some critical point where $e_{\max }$ eventually exceeds $e_{c} \approx 0.85$ the orbit hits the Earth surface within a certain period of time.

Area \#I is therefore where we observe the test orbits terminate in less than 200 years due to impact. We mark this area in accordance with the $e_{\max }$ contour in Fig. 1 and also the lifetime contour in Tang and Liu (2015). Compared to Area \#I, what is special about Area \#II is that quite a few special orbits in Area \#II do not terminate within 200 years, which can be found in both the lifetime and $e_{\max }$ contours. Fig. 4 (right frame) shows the lifetime and $e_{\max }$ along the $\mathbf{v} \mathbf{1}$ profile.

To demonstrate the dynamics, the tests are selected along the $\mathbf{v} \mathbf{1}$ profile. Fig. 12 shows the time series of $q_{10}$ and eccentricity for selected tests in Areas \#I $\left(\Omega_{0}=120^{\circ}, 130^{\circ}, 140^{\circ}\right)$ and \#II $\left(\Omega_{0}=170^{\circ}, 180^{\circ}, 190^{\circ}\right)$. The reason to the difference between the orbit lifetime and $e_{\max }$ in Areas \#I and \#II is that for the special orbit in Area \#II the $q_{10}$ argument somehow manages to stay around $q_{10}=0$ although it may not exhibit pure periodicity. With the constrained motion around $q_{10}=0$, the maximum eccentricity in the selected tests is around 0.7 , below $e_{c} \approx 0.85$.

One may wonder that starting around $\Omega_{0}=\pi$, the mean inclination should be several degrees larger than the theoretical resonance inclination (Table 1), and thus it is unlikely that they would librate. It is therefore worth to mention that the resonance inclination is theoretically obtained assuming 

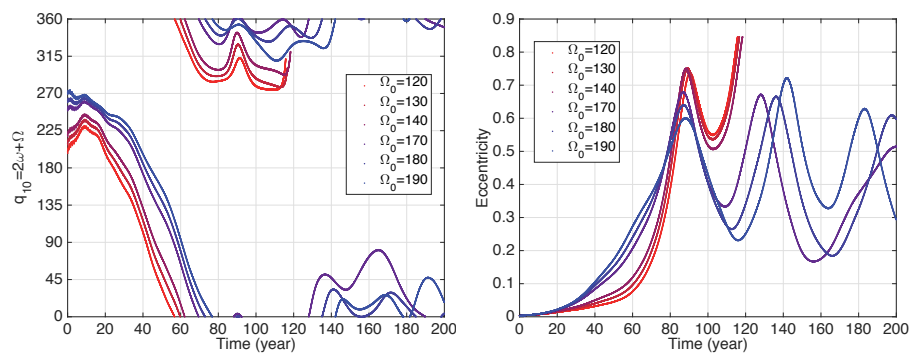

Figure 12: Time series of $q_{10}$ (left) and eccentricity (right) of selected cases in Areas \#I and \#II along profiles v1.
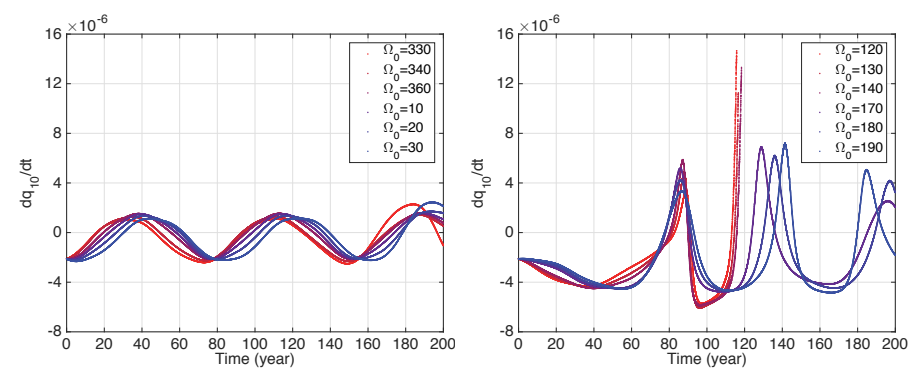

Figure 13: Time series of $\dot{q}_{10}$ (normalized) for tests around $\Omega_{0}=0$ (left) and tests in Areas \#I and \#II, all of which belong to profile $\mathbf{v} \mathbf{1}$. The acceleration $\dot{q}_{10}$ is computed using the 1-dof equations of motion.

a small eccentricity, when the eccentricity is large it is natural that some different inclination is required to satisfy the equilibrium condition $\dot{q}_{10}=0$. This is the reason to the longer lifetime in Area \#II. To confirm, we compare the acceleration of the argument $q_{10}$ in different areas selected along the $\mathbf{v} \mathbf{1}$ profile, including \#I, \#II and the vicinity of $\Omega_{0}=0$. The accelerations are computed using the 1-dof equation of motion $\dot{q}_{10}$ and are plotted in Fig. 13. In the vicinity of $\Omega_{0}=0$, we see that $\dot{q}_{10}$ varies periodically around 0 and the normalized magnitude of the acceleration does not exceed $2 \times 10^{-6}$ and in Area \#II the acceleration is still more or less periodic with a normalized amplitude of around $(4 \sim 6) \times 10^{-6}$. Compared to the former two areas, the $q_{10}$ acceleration in Area \#I is distinct in terms that after about 110 years it increases all the way to around $1.4 \times 10^{-5}$ until the lifetime terminates. This is what distinguishes Area \#I from \#II. 


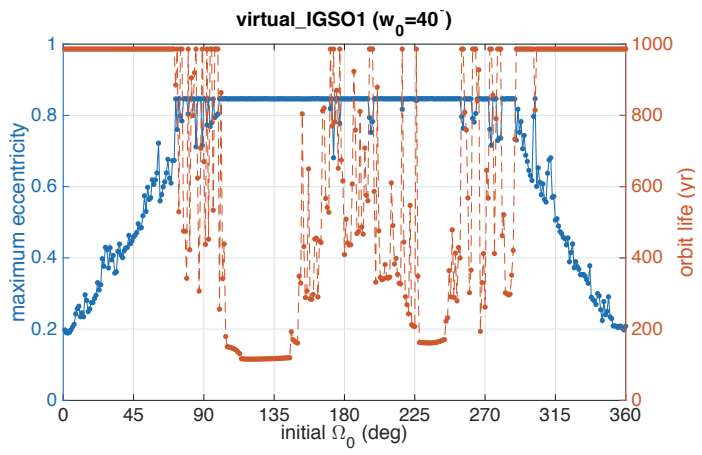

Figure 14: The maximum eccentricity (left $y$-axis of blue line with dots) and the orbital lifetime (right $y$-axis of red dash line with dots) with respect to $\Omega_{0}$, computed along the profile of $\omega_{0}=40^{\circ}$ in the scope of about 1000 years. Like the 200-year results, the equations of motion are integrated using the full force models.

\subsection{Secular resonance in 1000 years}

In the previous section, we investigated in depth the 200 year ephemerides over the $\omega_{0} \times \Omega_{0}$ grid. Secular resonances of various arguments play an important role in shaping the contours. However, we see that many of the secular resonances cannot last longer than a few decades, so it is interesting to see how the lifetime and $e_{\max }$ change with the initial phase $\Omega_{0}$ in a longer time span.

To that end, we choose the $\mathbf{v} \mathbf{1}$ profile again and numerically propagate the orbits for 1000 years using the same model as in Section 2.2. We mention that due to the time span of the DE406 ephemeris the orbit can only be propagated for approximately 986.2 years starting from the given initial epochs. Nevertheless it serves well enough for us to understand the dynamics over about a millennium.

Fig. 14 shows the dependence of the orbit lifetime and $e_{\max }$ on the initial phase $\Omega_{0}$. It is obtained generally in the same way as for the right frame of Fig. 4 except that it is propagated for a millennium.

The first impression, compared to the 200-year figure, is that the specific patterns such as points $\boldsymbol{a}, \boldsymbol{e}$ and Areas \#II, \#III now no longer exist. Fig. 14 suggests that in the scope of 1000 years, the secular resonances of the arguments $q_{0}, q_{2}$ and $q_{9}$ that shape the stripe for a few decades are simply transient. In a longer time span, they are much less important than in just 200 years. The equilibrium state of $q_{10}$ in Area \#II is able to reduce the $e_{\max }$ value, and it indeed increases the orbit lifetime, from a little more than 100 years in the neighboring Area \#I to a few centuries in Area \#II. However, the 
long-term tendency is that the equilibrium around $\Omega_{0}=\pi$ sustained under large eccentricity would still gradually fail.

The dependence of $e_{\max }$ and orbit lifetime on the initial phase $\Omega_{0}$ now looks much simpler. As is already seen in the 200-year analysis, this relation clearly suggests the important role that the $q_{10}$ argument could play in the long-term evolution and it is easy to be verified by investigating the $q_{10}$ series over the millennium. Along the $\mathbf{v} \mathbf{1}$ profile, we investigate the time series for $\Omega_{0} \in\left\{1^{\circ}, 11^{\circ}, 21^{\circ}, 31^{\circ}, 41^{\circ}, 51^{\circ}\right\}$, as well as those corresponding to the transient secular resonances $\Omega_{0} \in\left\{67^{\circ}(\boldsymbol{a}), 276^{\circ}(\mathbf{I I I}), 351^{\circ}(\boldsymbol{e})\right\}$. Those figures are provided in the supplementary materials ${ }^{6}$.

It can be concluded from the millennium ephemerides that for the BDS IGSO configuration over such a long period of time, only the $q_{10}$ argument is possibly able to remain in the state of libration and thus contributes to restraining the eccentricity, while other resonance arguments can last no longer than a few decades and would have no major influence for the centuryor millennium-level orbit evolution.

\subsection{Orbit resonance under large eccentricity}

Regarding the long-term orbital evolution of BDS IGSO satellites, as well as the disposal strategy, we investigate the orbit resonance when the eccentricity gets large under the secular resonance.

Since the BDS IGSO satellites are in the geosynchronous orbit, the tesseral terms like $J_{2,2}, J_{3,1}$ would cause orbit resonance between the satellites' mean motion and the Earth rotation. This resonance effect can be represented using the $a-l$ phase diagram, where $l=\Omega+\omega+M-S_{G}\left(S_{G}\right.$ is the Greenwich sidereal time) is the elongation between the satellite and the prime meridian (Zhao et al., 2015). We mention the orbit resonance in the mean motion here because the large eccentricity induced by the secular resonance can affect the orbit resonance. With the increasing eccentricity, the short-period term of the semi-major axis increases at the perigee ${ }^{7}$.

When the short-period term of the semi-major axis is sufficiently large, the motion around the stable equilibrium in the $a-l$ diagram is possible

\footnotetext{
${ }^{6}$ Specifically, the supplementary materials include the millennium series of $q_{10}$ and $e$ for $\Omega_{0} \in\left\{1^{\circ}, 11^{\circ}, 21^{\circ}, 31^{\circ}, 41^{\circ}, 51^{\circ}\right\}$, and those of $q_{0}, q_{2}, q_{9}, q_{10}$ and $e$ for $\Omega_{0} \in$ $\left\{67^{\circ}, 276^{\circ}, 351^{\circ}\right\}$. These high-resolution figures are too large to be included in the manuscript.

${ }^{7}$ This is demonstrated in the additional figures in the supplementary material.
} 

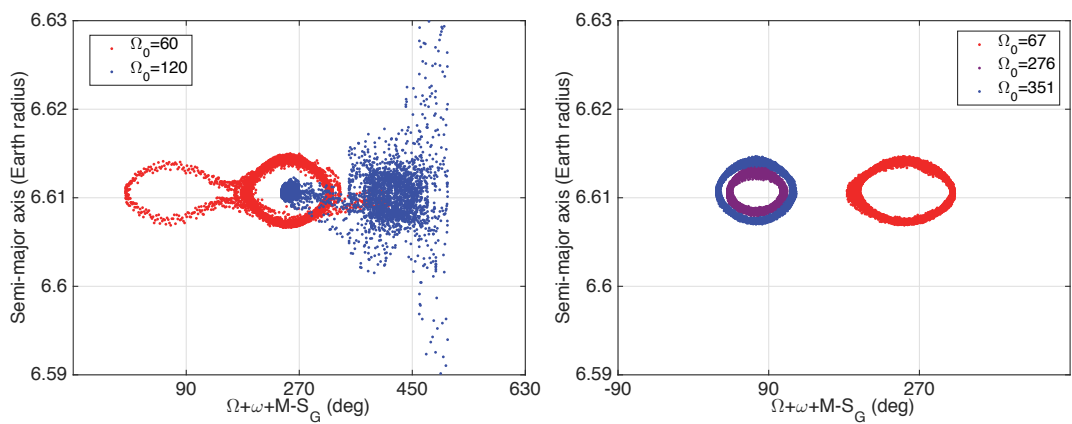

Figure 15: $a-l$ phase diagrams at $\Omega_{0}=60^{\circ}, 120^{\circ}$ (left) and at $\Omega_{0}=67^{\circ}(\boldsymbol{a}), 276^{\circ}$ (III), $351^{\circ}(\boldsymbol{e})$ (right), all selected along $\mathbf{v} \mathbf{1}$.

to transit to other equilibria. To demonstrate the effect, we select several tests along the $\mathbf{v} \mathbf{1}$ profile and plot in Fig. 15 the $a-l$ phase diagrams for $\Omega=60^{\circ}, 120^{\circ}$ where $e$ becomes large and also for $\Omega=67^{\circ}(\boldsymbol{a}), 276^{\circ}$ (III) and $351^{\circ}(\boldsymbol{e})$ where the eccentricity is effected suppressed in 200 years. As long as the eccentricity remains small, the $a-l$ resonance can steadily librate around one equilibrium without transiting to other ones.

We have seen that a major contribution to the transition of the orbit resonance is the eccentricity, nevertheless other factors can contribute as well. We select a few tests in the lower and upper parts of $\mathbf{v} \mathbf{1}$, whose $a-l$ phase diagrams and temporal $e$ series are provided in the supplementary materials. For the lower part we find three 'switch locations', each of which corresponds to the switch of $a-l$ resonance from 'transit' to 'non-transit' or the other way round. It is shown that in different cases, the eccentricity values starting the equilibrium transition are not necessarily the same. It becomes more evident if we observe the upper part of $\mathbf{v} \mathbf{1}$. For all selected tests, even the one with $e_{\max } \approx 0.5$, the $a-l$ resonance always librate around the same equilibrium at about $l=\pi / 2$.

This effect calls for attention regarding disposing the BDS IGSO satellites. From the general point of view, transiting between the equilibria suggests chaotic motions. It contributes to the complexity of the interaction between its own orbit and the orbit of other operational satellites and also to the decay of the decommissioned satellite. However, if the disposal of BDS IGSO satellite follows the recommendation of the Inter-Agency Space Debris Coordination Committee (IADC) (see e.g. Anselmo and Pardini (2008)), the orbit altitude will be raised by $250 \mathrm{~km}$ at the end of life. With this maneuver, 
the 1:1 orbit resonance can be avoided.

It should be clarified that the discussion in this subsection is about the 1:1 orbit resonance, which results from the proper value of the semi-major axis and can be rendered ineffective by an appropriate altitude-changing maneuver. However, to consider a complete avoidance of GEO/IGSO region, the orbit eccentricity is also a major concern. Tests and discussions in the prior sections have shown that complicated investigations are needed to determine whether or how such disposal strategy can be found to make sure the decommissioned BDS IGSO satellite does not invade the GEO/IGSO region.

\section{Conclusion}

This paper discusses the long-term orbit evolution of the BDS IGSO. We first draw some preliminary results using the simplified theoretical models. These results show the possible equilibria and motion patterns in the BDS IGSO evolution and prepare us for analyzing the following numerical results.

For the actual scenario, we present the long-term evolutions of the BDS IGSO configurations over the complete $\left(\omega_{0} \times \Omega_{0}\right)=(2 \pi \times 2 \pi)$ grid, which are numerically propagated using the real force models. These numerical results are generally consistent with the theoretical ones, and the models serve well for us to understand the underlying dynamics of the BDS IGSO.

For the secular resonances, we investigate the long-term evolution in the scope of both 200 years and 1000 years. It is shown that in the first few decades the resonance arguments of $q_{0}, q_{2}$ and $q_{9}$ are able to dominate the system if the equilibrium condition is satisfied and the initial phase is appropriate. These resonances are highlighted in a specific stripe pattern in the $\omega_{0}-\Omega_{0}-e_{\max }$ contour. In the presence of the resonance, the eccentricity can be suppressed at small values. However, from the millennium-level perspective, it appears that such resonances are trivial and these specific patterns no longer exist.

On the other hand, the secular resonance of the $q_{10}$ argument is prominent, especially for those starting near $\Omega_{0}=0$. It exists and is usually able to last for as long as a millennium. In an approximate $20^{\circ}$ neighborhood of $\Omega_{0}=0$, the system is able to enter the equilibrium state of the $q_{10}$ argument and as a result the eccentricity over 1000 years would never exceed 0.3 (reference to the supplementary materials recommended). For the best case where the system starts closest to the resonance inclination of $q_{10}$ (in the vicinity of 
$\Omega_{0}=0$ or the point $\boldsymbol{e}$ ), the 200 -year-level $e_{\max }$ can be as small as 0.01 , while the millennium-level $e_{\max }$ is approximately 0.15 .

Regarding the disposal strategies for the BDS IGSO, it is one option to raise the satellite to the graveyard. If we can appropriately adjust the orbital elements, for example the (osculating) inclination and the time of orbit insertion (that determines $\Omega$ without having to change the sub-satellite geographical longitude if necessary), it is possible that we can put the satellite closer to the $q_{10}$ resonance that, according to the 1-dof model and the supplementary figures, could further suppress the $e_{\max }$ values. In the case that the secular resonance of $q_{10}$ dominates, it could leave the option still open to dispose the decommissioned BDS IGSO satellite by raising it to the graveyard orbit. Nevertheless, it may require accurate orbit design and insertion to make sure the disposal orbit should keep a safe distance from the geosynchronous orbit. Another possible option to take advantage of the increasing eccentricity is to inject the satellite to a fast-decay orbit that re-enters the atmosphere. However, for the BDS IGSO, the result over the $\Omega_{0} \times \omega_{0}$ grid suggests that even the fastest decay still takes around 120 years (Tang and Liu, 2015).

When the eccentricity increases to large values, we show that the commonly known 1:1 orbit resonance can be affected, in terms that the $a-l$ resonance transits in longitude from one equilibrium to another. This suggests that if the decommissioned BDS IGSO satellite (e.g. for \#1, \#3 or \#5) is left uncontrolled in orbit, it may wander in longitude among different equilibrium positions. One possible way to get rid of the 1:1 resonance and to mitigate the chaotic drift in the longitude is simply to follow the IADC recommendation and to raise the orbit altitude with the disposal maneuver.

Last but not least, although we have employed the resonance arguments to explain how the secular resonance shapes the lfietime/ $e_{\max }$ contours in the $\omega_{0} \times \Omega_{0}$ grid, there are still further questions that can be investigated, such as the reason for which the resonance arguments have different roles in the long-term evolution. These problems would be further investigated in our future work.

\section{Acknowledgement}

The authors thank Haowen Cheng for helpful comments on the manuscript. The authors also appreciate the anonymous reviewers for the insightful comments that help us to substantially improve the manuscript. Xiyun Hou 
is supported by the National Science Foundation of China (11322330) and the National Basic Research Program of China (2013CB834100). The initial elements of the 5 BDS IGSO satellites are converted from the Two-Line Elements downloaded from celestrak.com.

Alessi E.M., Rossi A., Valsecchi, G.B., Anselmo, L., Pardini, C., Colombo, C., Lewis, H.G., Daquin, J., Deleflie, F., Vasile, M., Zuiani, F., Merz, K., 2014, Effectiveness of GNSS disposal strategies, Acta Astronautica, 99, 292-302

Anselmo, L., Pardini, C., 2008, Space debris mitigation in geosynchronous orbit, Adv. Space Res., 41(7), 1091-1099

Chao, C.C., Gick, R.A., 2004, Long-term evolution of navigation satellite orbits: GPS/GLONASS/GALILEO, Adv. Space Res., 34(5), 1221-1226

Fehlberg, E., 1968, Classical Fifth-, Sixth-, Seventh-, and Eighth-order Runge-Kutta Formulas with Stepsize Control, NASA Technical Report, TR R-287

Hughes, S., 1980, Earth Satellite Orbits with Resonant Lunisolar Perturbations. I. Resonances Dependent Only on Inclination, Proc. R. Soc. Lond. A, 372, 243-264

Jin, S.G., Jin, R., Li, D., 2016, Assessment of BeiDou differential code bias variations from multi-GNSS network observations, Ann. Geophys., 34(2), 259-269

Kozai, Y., 1959, The motion of a close earth satellite, Astron. J., 64, 367-377

Kozai, Y., 1973, A New Method to Compute Lunisolar Perturbations in Satellite Motions, Smithsonian Astrophys. Obs. Sepc. Rep. \#349, 1-27

Liu, L., Liao, X., Zhao, C., Wang, C., 1994, Application of symplectic algorithm to dynamical astronomy III, Chin. Astron. Astrophys., 35(1), 323339

Liu, L., 2000, Orbit theory for spacecraft, National Defense Industry Press, Beijing (in Chinese)

Murray, C.D., Dermott, S.F., 1999, Solar System Dynamics, Cambridge University Press 
Pardini, C., Anselmo, L., 2012, Post-disposal orbital evolution of satellites and upper stages used by the GPS and GLONASS navigation constellations: The long-term impact on the Medium Earth Orbit environment, Acta Astronaut., 77, 109-117

Rosengren, A.J., Alessi, E.M., Rossi, A., Valsecchi, G.B., 2015, Chaos in navigation satellite orbits caused by the perturbed motion of the Moon, Mon. Not. R. Astron. Soc., 449, 3522-3526

Rossi, A., 2008, Resonant dynamics of Medium Earth Orbits: space debris issues, Celest. Mech. Dyn. Astr., 100(4), 267-286

Stefanelli, L., Metris, G., 2015, Solar gravitational perturbations on the dynamics of MEO: Increase of the eccentricity due to resonances, Adv. Space Res., 55(7), 1855-1867

Tang, J., Liu, L., Cheng, H., 2012, An extended discussion on the DoubleStar orbits, IAC-11.C1.3.4, in Proc. 62nd International Astronautical Congress (Vol.6)., 4772-4778

Tang, J., Liu, L., 2015, Disposal strategy for the geosynchronous orbits of the Beidou Navigation Satellite System, IAC-14.A6.9.6, in Proc. 64th International Astronautical Congress (Vol.3), Paris, 1791-1795

Zhao, C.Y., Zhang, M.J., Wang, H.B., Xiong, J.N., Zhu, T.L., Zhang, W., 2015, Analysis on the long-term dynamical evolution of the inclined geosynchronous orbits in the Chinese BeiDou navigation system, Adv. Space Res. 56(3), 377-387 